\title{
IMPACTO DOS REGIMES DE MANEJO FLORESTAL SOBRE USO E CONSERVAÇÃO DOS RECURSOS FLORESTAIS E RENDIMENTOS DOS INTERVENIENTES EM SAVANE, MOÇAMBIQUE
}

\author{
Mário Paulo Falcão ${ }^{1}$, Dartagnan Baggio Emerenciano ${ }^{2}$ \\ ${ }^{1}$ Eng. Florestal, Dr., Depto. de Engenharia Florestal, Universidade Eduardo Mondlane, Maputo, Moçambique - \\ mariopaulofalcao@hotmail.com \\ ${ }^{2}$ Eng. Florestal, Dr., Depto. de Ciências Florestais, UFPR, Curitiba, PR, Brasil - dartagnanbaggio@ gmail.com
}

Recebido para publicação: 09/06/2010 - Aceito para publicação: 13/07/2011

\begin{abstract}
Resumo
As altas taxas de desmatamento em Moçambique, estimadas em 0,24\% ao ano, associadas à degradação da terra trouxeram mudanças institucionais na procura de políticas e estratégias para manejar os recursos florestais. Regimes de manejo florestal e políticas que satisfaçam as necessidades dos vários intervenientes e garantam o uso sustentável dos recursos florestais são um complexo para ser analisado. Este estudo de caso realizado no distrito do Dondo, localidade de Savane, analisa o impacto das várias alternativas de regime de manejo florestal sobre os rendimentos dos usuários e a conservação da floresta de miombo. Modelos dinâmicos de sistemas baseados na teoria de jogos foram desenvolvidos e implementados com base no software POWERSIM. Os resultados mostram que regimes de manejo de comando (centralizados), incorporando aspectos sociais ou sociais e ambientais são potencialmente mais benéficos para o setor familiar que o regime de licença simples ou não cooperativo. O regime de licença é a opção mais benéfica de manejo florestal para o setor privado.

Palavras-chave: Teoria de jogos; modelo dinâmico sistemático.
\end{abstract}

\begin{abstract}
Impact of forest management regimes of forest resource use, conservation and income of stakeholders in Savane, Mozambique. Higher deforestation rates in Mozambique, estimated as $0.24 \%$ per annum, associated with land degradation brought institutional changes in the search for adequate policies and strategies for the management of its natural resources. Forest management regimes and policies that satisfies the needs of several stakeholders and guarantee sustainable use of forest resources, is a complex to be analysed. A case study was carried out in Dondo district, Savane locality, to analyse the impact of alternative forest management regimes on the well being of stakeholders and conservation of the miombo woodlands. Dynamic game theoretic models based o game theory were developed and implemented with software POWERSIM. This study shows that regulated forest management regimes, incorporating social concerns or social and environmental concerns, are potentially more beneficial to the household sector that the open access regime. The open access is more beneficial regime to the private sector.

Keywords: Forest management regimes; game theory; stakeholders; dynamic game theoretic model.
\end{abstract}

\section{INTRODUÇÃO}

Os recursos florestais desempenham um papel importante na economia moçambicana, além do que são importantes para a sobrevivência e segurança alimentar da população rural desse país. As altas taxas de desmatamento, estimadas em $0,24 \%$ ao ano, associadas à degradação da terra têm estimulado a reavaliação das instituições e políticas que orientam a exploração e conservação dos recursos florestais.

Segundo o Banco Mundial (2001), qualquer estratégia sustentável da redução de pobreza deve assegurar o acesso aos recursos naturais pelos pobres e ao mesmo tempo criar condições que permitam às comunidades manejá-los de forma sustentável. Os projetos de manejo conjunto ou manejo comunitário já estão assumindo um papel importante nos programas de manejo florestal em muitos países da África subsaariana. 
O primeiro projeto de manejo dos recursos naturais em Moçambique foi implementado em 1994 na comunidade de Bawa, província de Tete, localizada na fronteira com o Zimbabwe (MURPHREE, 1997; WILY; MBAYA, 2001). Esse projeto, conhecido como 'Tchuma-Tchato', foi desenhado com o objetivo de resolver os conflitos existentes entre as comunidades, a fauna bravia e o operador de safári. Um dos objetivos foi controlar ou minimizar a caça furtiva, que era realizada pelos residentes locais, zimbabweianos e zambianos, a polícia e funcionários governamentais (NHANTUMBO, 2000). O relativo sucesso desse programa encorajou o rápido surgimento de projetos novos no país. Destaque-se que, 4 anos após o estabelecimento do projeto Tchuma-Tchato, cerca de 40 projetos foram implantados por diferentes instituições governamentais, locais e internacionais, com apoios financeiros dos doadores (NHANTUMBO, 2000; ANSTEY, 2001; WILY; MBAYA, 2001).

Exemplos na região da Comunidade de Desenvolvimento para a África Austral (SADC) incluem o CAMPFIRE (programa de manejo de áreas comunitárias para recursos naturais) no Zimbabwe, que envolve as comunidades locais no manejo de fauna bravia. O programa ADMADE (Projeto Administrativo de Gestão para Manejo de Áreas de Caça) da Zâmbia foi outra iniciativa de manejo da fauna bravia, com a participação das comunidades locais (FOREST TREES AND PEOPLE NEWSLETTER, 1991) e comitês de vilas de manejo dos recursos naturais no Malawi.

Outras iniciativas incluem também Confiança, Conservação e Associações ("Trusts, Conservancies and Associations") encontrados em Botswana, Namíbia e em algumas vilas na Tanzânia (por exemplo, Duru-Haitemba).

Essa mudança de políticas de estrito controle pelo estado é um resultado dos ineficientes instrumentos de regulação, resultando em altos custos e conflitos entre os objetivos dos vários intervenientes. Os objetivos múltiplos a serem alcançados incluem produção industrial de madeira, fornecimento de bens de sobrevivência para as comunidades locais (material de construção, combustíveis lenhosos e pastos) e fornecimento de serviços (fornecimento sustentável de água potável, amenização climática e controle da erosão dos solos). Esses serviços são essencialmente obtidos indiretamente através da conservação. $O$ fato de diferentes intervenientes terem objetivos conflituosos sobre os recursos florestais levou à criação de estratégias de manejo cooperativo, que são uma tarefa complexa. O presente artigo demonstra como modelos dinâmicos de sistemas ("system dynamics models") podem ser utilizados para avaliar diferentes alternativas de regimes de manejo onde estão envolvidos múltiplos intervenientes, empregando dados recolhidos em Savane, no distrito do Dondo ${ }^{1}$.

Os modelos baseados na teoria de jogos são promissores para análise de assuntos de manejo onde vários intervenientes estão envolvidos com objetivos conflituosos. White (2000) faz uma revisão detalhada de análises da teoria de jogos para recursos de propriedade comum. Com exceção da área de pesca, a aplicação de modelos baseados na teoria de jogos para recursos renováveis é limitada. McCarthy (2000) desenvolveu um modelo teórico baseado na teoria de jogos para analisar os efeitos de risco da produção de gado no uso de terras de pastos livres. Os seus resultados de estatísticas comparativas mostram que, sob regime cooperativo, criadores são melhores em termos de bem-estar e produção com diminuição do risco de produção.

Kant e Nautiyal (1993), usando a aproximação de jogos de monopólio bilateral, mostraram a necessidade de as comunidades partilharem os benefícios dos recursos florestais para manterem os seus interesses na cooperação com o proprietário da floresta no manejo florestal conjunto. Esse modelo, contudo, é limitado, pelo fato de não levar em consideração outras formas de emprego para as comunidades locais (agricultura e emprego fora das machambas/roças). Cardenas et al. (2000) investigaram os efeitos de instituições externas, como regras e regulamentos impostos de fora da comunidade no comportamento sob um arranjo experimental. Os resultados desses autores mostraram que políticas ambientais locais que são moderadamente aplicadas são ineficazes e podem prejudicar quando comparadas à permissão de que indivíduos coletivamente possam confrontar o dilema ambiental local sem intervenção.

\footnotetext{
${ }^{1}$ Mlay, G. I.; Falcão, M.; Nhantumbo, I. \& Kowero, G. (2003). Policy impact on woodland resource management, use and conservation in Mozambique: Case study of selected sites in Dondo, Nhamatanda, Gondola and Manica Districts. In G. Kowero, B. M. Campbell \& R. Sumaila (Eds). Policies and governance structures in woodlands of Southern Africa, CIFOR, Bogor.
} 
Este estudo teve como objetivo avaliar o impacto socioeconômico e ambiental do uso da floresta de miombo e identificar as melhores opções de manejo. O estudo teve os seguintes objetivos específicos: identificar o regime de manejo mais apropriado e avaliar os impactos socioeconômicos e ambientais.

Com este estudo, testou-se a hipótese de que a gestão comunitária de recursos florestais promove a melhor conservação dos recursos, embora leve à redução dos benefícios econômicos do setor privado e das comunidades.

\section{MATERIAIS E MÉTODOS}

Nesta seção, apresenta-se o modelo conceitual de tomada de decisões pelos intervenientes e a identificação dos possíveis cenários de manejo a serem aplicados. Também são apresentados os modelos de otimização baseados na teoria de jogos, refletindo os cenários de manejo anteriormente identificados. Na última parte apresentam-se os dados necessários para a utilização do modelo.

\section{A moldura conceitual}

Neste trabalho, foi utilizado o modelo dinâmico de sistemas ("system dynamics model") baseado na teoria de jogos. O modelo conceitual adaptado consta, detalhado, em Sumaila et al. (2001). Três grupos de intervenientes foram identificados: o governo, o setor privado e a comunidade local. $\mathrm{O}$ interesse do governo em floresta de miombo baseia-se na maximização dos benefícios para a sociedade, que incluem proteção ambiental (biodiversidade, proteção de áreas de retenção, mitigação da degradação da terra e mudanças climáticas) e benefícios econômicos diretos e sociais (por exemplo, preservação de áreas para habitação nas zonas rurais). O objetivo das comunidades na floresta de miombo é baseado nos benefícios que podem obter a partir da exploração dos produtos madeireiros e não madeireiros para consumo e comercialização, enquanto que o setor privado está interessado na obtenção de rendimentos, derivados das atividades de exploração de toras. Sob a legislação moçambicana, a terra pertence ao estado (proprietário), enquanto as comunidades e o setor privado se beneficiam do seu uso com base em regulamentos estabelecidos pelo governo. A falta de capacidade para fazer cumprir os regulamentos tem como resultado uma exploração desordenada da floresta de miombo. Isso tem potencializado conflitos entre os intervenientes, devido à divergência de objetivos. Enquanto o setor privado realiza exploração de toras de modo seletivo, o setor familiar realiza atividades de exploração não seletiva, levando à destruição de madeira com alto valor comercial. Ademais, exploração sob concessão impede o acesso legal das comunidades a essas áreas e, na ausência de capacidade de implementação da legislação, essas áreas continuarão a ser exploradas pelas comunidades como se estivessem num regime de acesso aberto, levando à perda de espécies madeireiras de alto valor comercial, que são legalmente alocadas ao proprietário da concessão. Esses conflitos servem de base para se considerar a cooperação entre o setor privado e as comunidades.

\section{Processo de decisão dos setores familiar e privado}

As decisões seguindo a teoria de jogos estão sumarizadas na figura 1. Assumiu-se que em qualquer momento "t" existe uma área fixa de floresta de miombo $\left(\mathrm{N}_{\mathrm{t}}\right)$ disponível para as comunidades, na qual a área $\left(\mathrm{N}_{\mathrm{a}}\right)$ está sob agricultura e $\left(\mathrm{N}_{\mathrm{m}}\right)$ é a área remanescente de floresta miombo. Os setores familiar e privado têm de decidir sobre como utilizar a área $\left(\mathrm{N}_{\mathrm{m}}\right)$ hectares que está sob a forma de floresta de miombo. $\mathrm{O}$ processo de decisão do setor privado é um estágio envolvendo a determinação da área $\left(\mathrm{H}_{\mathrm{c}}\right)$ para exploração anual, de modo a maximizar a soma dos benefícios líquidos descontados. Os critérios utilizados no processo de decisão refletem o que aconteceu no campo.

No caso do setor familiar, o processo de decisão envolve três estágios. O critério para cada estágio de decisão é maximizar a utilidade no tempo. O primeiro estágio de decisão é feito sobre que quantidade da área presentemente sob forma de floresta de miombo deve ser usada $\left(\mathrm{H}_{\mathrm{h}}\right)$, de maneira a maximizar a sua utilização no tempo. O segundo estágio de decisão é feito em relação a quanto da área de floresta de miombo será desmatada pelo setor familiar para abertura de machambas/roças $\left(\mathrm{H}_{\mathrm{a}}\right)$ e quanto será usada para a exploração de produtos madeireiros $\left(\mathrm{H}_{\mathrm{m}}\right)$. Para a área retida para exploração de produtos madeireiros, o terceiro estágio envolve a alocação entre estacas, $\mathrm{H}_{\mathrm{po}}$, e combustível lenhoso, $\mathrm{H}_{\mathrm{fw}}$. $\mathrm{O}$ processo de alocação é conduzido pelos preços relativos. 


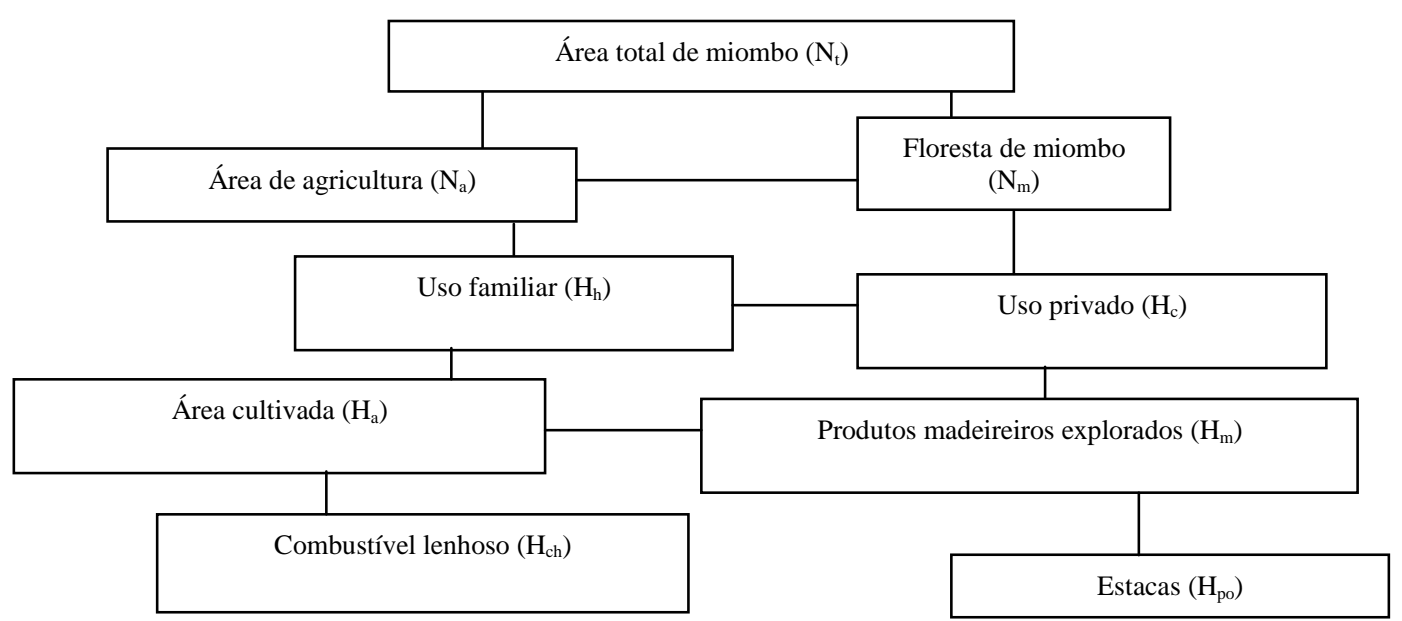

Figura 1. Etapas de decisão pelos setores privado e familiar.

Figure 1. Steps in decision making by the household and commercial sectors.

\section{Dinâmica da floresta}

A ecologia do miombo foi representada pela equação (1) de regeneração, na qual a taxa de regeneração foi assumida como constante ao longo do ano, e o estoque após a intervenção está apresentado na equação (2):

$$
\begin{aligned}
& \mathrm{R}_{\mathrm{t}}=\mathrm{K}_{\mathrm{t}} \\
& \mathrm{N}_{\mathrm{t}}=\mathrm{sN}_{\mathrm{t}-1}+\mathrm{R}_{\mathrm{t}}-\mathrm{H}_{\mathrm{c}, \mathrm{t}}-\mathrm{H}_{\mathrm{h}, \mathrm{t}}
\end{aligned}
$$

em que: $\mathrm{N}_{\mathrm{o}}=$ estoque inicial da floresta de miombo (quando $\mathrm{t}=1$ );

$$
\mathrm{s}=\text { taxa de sobrevivência. }
$$

Para a implementação do modelo, foi utilizada a área de floresta de miombo (ha) e o volume médio por hectare $\left(\mathrm{m}^{3} / \mathrm{ha}\right)$.

$\mathrm{O}$ volume de produtos de miombo a ser explorado pelo setor familiar depende do volume em pé da floresta $(\mathrm{N})$ e da mão de obra alocada nessa atividade $\left(\mathrm{L}_{\mathrm{m}}\right)$. A função de Cobb-Douglass representada na equação (3) foi usada para descrever a funcionalidade dessa relação.

$$
\mathrm{H}_{\mathrm{m}}=\mathrm{q}_{\mathrm{h}} \mathrm{N}^{\mu} \mathrm{L}_{\mathrm{m}}^{\psi} \quad 0<\mu<1,0<\psi<1
$$

em que: $\mathrm{q}_{\mathrm{h}}=\mathrm{o}$ parâmetro de eficiência familiar;

$\mu$ e $\psi=$ elasticidades parciais de produção.

\section{Os modelos da teoria de jogos}

Três principais modelos de teoria de jogos são utilizados, nomeadamente, o modelo centralizado (modelo comando), o modelo cooperativo e o modelo não cooperativo (livre acesso). Para obter informações mais detalhadas sobre os componentes dos modelos, ver Falcão et al. (2007).

\section{Modelo centralizado ou de comando}

Esse modelo é aplicável para regimes de manejo que pressupõem a existência de um órgão regulador, que pode ser o governo central ou local, que detém a propriedade e toma decisões de forma centralizada. Nesse modelo, os benefícios líquidos da sociedade são maximizados por meio da seleção da quantidade de mão de obra $\left(\mathrm{L}_{\mathrm{t}}\right)$ a ser utilizada pelos setores privado e familiar em cada ano no período $t$ de simulação do modelo (equação 4). As tecnologias de exploração usadas por ambos os setores são mão de obra intensiva e, portanto, a quantidade de mão de obra define o volume de produtos madeireiros explorados por cada setor.

$$
\max _{\mathrm{L}} \sum_{\mathrm{T}=1}^{\mathrm{T}}\left[\mathrm{B}_{\mathrm{t}}\right] \rho_{\mathrm{t}-1}=\sum_{\mathrm{t}=1}^{\mathrm{T}} \rho_{\mathrm{t}-1}\left[\mathrm{~B}_{\mathrm{c}}\left(\mathrm{H}_{\mathrm{c}, \mathrm{t}}\right)+\mathrm{B}_{\mathrm{h}}\left(\mathrm{H}_{\mathrm{h}, \mathrm{t}}\right) \mathrm{B}_{\mathrm{s}}\left(\theta_{\mathrm{c}} \mathrm{H}_{\mathrm{c}, \mathrm{t}}, \theta_{\mathrm{h}, \mathrm{t}} \mathrm{H}_{\mathrm{h}, \mathrm{t}}\right)-\emptyset \mathrm{B}_{\mathrm{e}}\left(\mathrm{H}_{\mathrm{c}, \mathrm{t}}+\mathrm{H}_{\mathrm{h}, \mathrm{t}}\right)\right]
$$


Sujeito a: $N_{t}=s N_{t-1}+K_{t}-H_{c, t}-H_{h, t}$

$$
L_{t}=L_{m, t}+L_{a, t}+L_{n, t}+L_{o f, t}
$$

em que: $\mathrm{B}_{\mathrm{t}}=$ benefícios totais líquidos aos setores: familiar e comercial; $\mathrm{B}_{\mathrm{c}}=$ benefícios privados líquidos recebidos pelo setor comercial; $\mathrm{B}_{\mathrm{h}}=$ benefícios privados líquidos recebidos pelo setor familiar; $\mathrm{B}_{\mathrm{s}}=$ benefícios sociais; $\mathrm{B}_{\mathrm{e}}=$ benefícios de conservação do meio ambiente; $\rho=$ fator de desconto; $\mathrm{L}_{\mathrm{m}, \mathrm{t}}=$ mão de obra utilizada na exploração de produtos madeireiros no período $\mathrm{t} ; \mathrm{L}_{\mathrm{a}, \mathrm{t}}=$ mão de obra utilizada na agricultura no período $\mathrm{t} ; \mathrm{L}_{\mathrm{n}, \mathrm{t}}=$ mão de obra utilizada na conversão da floresta de miombo para agricultura no período $t ; \mathrm{L}_{\mathrm{of}, \mathrm{t}}=$ mão de obra utilizada na agricultura e em atividades fora da área florestal no período $\mathrm{t} ; \mathrm{s}=$ taxa de regeneração natural da floresta; $\theta_{\mathrm{c}}$ e $\theta_{\mathrm{h}}$ são pesos atribuídos aos setores, comercial e familiar, respectivamente, para representar as preferências da sociedade na exploração da floresta para fins sociais; $\phi=$ um parâmetro para capturar as preocupações da sociedade com o meio ambiente.

Dependendo dos valores atribuídos aos parâmetros $\theta_{\mathrm{c}}, \theta_{\mathrm{h}}$ e $\phi$, diferentes cenários de modelo centralizado podem ser avaliados. Neste artigo abordam-se três cenários de manejo centralizado. Um cenário no qual o regulador (governo central ou local) está interessado nos benefícios sociais, dando maior peso ao setor familiar, mas dentro dos limites aceitáveis de proteção do meio ambiente $\left(\theta_{\mathrm{c}}=0, \theta_{\mathrm{h}}=1 \mathrm{e} \phi=1\right)$. Esse cenário simula a conservação de florestas naturais com exploração limitada ou controlada, como em áreas florestais de retenção de água. Outro cenário no qual só os benefícios ambientais são considerados $\left(\theta_{\mathrm{c}}=0, \theta_{\mathrm{h}}=0 \mathrm{e} \phi=1\right)$. Este cenário representa estritamente a conservação da biodiversidade que usualmente é encontrada em áreas protegidas para biodiversidade, como locais de herança. E um cenário no qual somente os benefícios sociais são considerados e em favor do setor familiar $\left(\theta_{\mathrm{c}}=0, \theta_{\mathrm{h}}=1 \mathrm{e} \phi=0\right)$. O último cenário é largamente encontrado nas regiões de muita floresta. Algumas áreas estão completamente sob regime de acesso livre, enquanto outras estão tecnicamente sob controle do governo, mas devido a poucos recursos para o seu manejo essas florestas tem se tornado de acesso livre.

\section{Modelo cooperativo}

Nesse modelo é assumido que os setores privado e familiar têm incentivos para cooperar através da maximização conjunta dos benefícios líquidos descontados (equação 5). O modelo cooperativo simula a situação na qual as comunidades rurais estão envolvidas no manejo dos recursos florestais.

$$
\max _{L_{h}, L_{c}} \sum_{t=1}^{T}\left[\alpha \rho_{c, t-1} B_{c, t}+(1-\alpha) \rho_{h, t-1} B_{h, t}\right]
$$

Sujeito a: $\quad 0 \leq \alpha \leq 1$

$$
\begin{aligned}
& \mathrm{N}_{\mathrm{t}}=\mathrm{sN}_{\mathrm{t}-1}+\mathrm{K}_{\mathrm{t}}-\mathrm{H}_{\mathrm{c}, \mathrm{t}}-\mathrm{H}_{\mathrm{h}, \mathrm{t}} \\
& \mathrm{L}_{\mathrm{t}}=\mathrm{L}_{\mathrm{m}, \mathrm{t}}+\mathrm{L}_{\mathrm{a}, \mathrm{t}}+\mathrm{L}_{\mathrm{n}, \mathrm{t}}+\mathrm{L}_{\mathrm{of}, \mathrm{t}}
\end{aligned}
$$

em que: $\alpha=$ parâmetro cujo valor reflete o peso relativo atribuído aos benefícios dos dois grupos de intervenientes ("stakeholders").

\section{Modelo não cooperativo}

Nesse modelo é assumido que os setores familiar e comercial operam de maneira independente nas florestas, sem levar em consideração os interesses dos outros intervenientes. Esse modelo simula a situação de acesso livre à floresta, que atualmente é o regime dominante de exploração da floresta de miombo em várias partes de Moçambique.

O constrangimento para o problema de maximização para cada interveniente é indicado a seguir:

\section{i. Setor familiar}

$$
\max _{L_{h}} \sum_{t-1}^{T} \rho_{h, t-1} B_{h, t}
$$

Sujeito a: $\quad \mathrm{N}_{\mathrm{t}}=\mathrm{sN}_{\mathrm{t}-1}+\mathrm{K}_{\mathrm{t}}-\mathrm{H}_{\mathrm{c}, \mathrm{t}}-\mathrm{H}_{\mathrm{h}, \mathrm{t}}$

$$
\mathrm{L}_{\mathrm{t}}=\mathrm{L}_{\mathrm{m}, \mathrm{t}}+\mathrm{L}_{\mathrm{a}, \mathrm{t}}+\mathrm{L}_{\mathrm{n}, \mathrm{t}}+\mathrm{L}_{\mathrm{of}, \mathrm{t}}
$$


ii. $\underline{\text { Setor privado }}$

$\max _{L_{c}} \sum_{t-1}^{T} \rho_{c, t-1} B_{c, t}$

Sujeito a: $\quad 0 \leq \alpha \leq \mathrm{N}_{\mathrm{t}}=\mathrm{sN}_{\mathrm{t}-1}+\mathrm{K}_{\mathrm{t}}-\mathrm{H}_{\mathrm{c}, \mathrm{t}}-\mathrm{H}_{\mathrm{h}, \mathrm{t}}$

$\mathrm{L}_{\mathrm{t}}=\mathrm{L}_{\mathrm{m}, \mathrm{t}}+\mathrm{L}_{\mathrm{a}, \mathrm{t}}+\mathrm{L}_{\mathrm{n}, \mathrm{t}}+\mathrm{L}_{\mathrm{of}, \mathrm{t}}$

A simulação dos modelos foi baseada na aproximação numérica similar à utilizada por Sumaila (1995), usando "system dynamics simulation package Powersim". Os três modelos foram aplicados com base na introdução dos multiplicadores modificados de Lagrange e aplicando "non-smooth convex optimisation" (FLÅM, 1993). O programa Powersim foi desenvolvido pelo "ModellData AS" em Bergen, Noruega.

\section{Dados utilizados}

Os dados usados nos modelos básicos estão na tabela 1 a seguir. Esses dados foram obtidos a partir do trabalho de campo realizado no ano de 2005 e suplementados com informação de fontes secundárias.

Tabela 1. Dados básicos utilizados nos modelos.

Table 1. Basic data used to run the models.

\begin{tabular}{|c|c|c|}
\hline Item & Unidades & Valor \\
\hline Tamanho médio da machamba/roça & ha & 2,6 \\
\hline Fator de desconto $(\rho)$ & & {$[0,909 ; 0,89]$} \\
\hline Parâmetro de eficiência da mão de obra do setor familiar $\left(\mathrm{q}_{\mathrm{h}}\right)$ & & {$[0,1 ; 0,083]$} \\
\hline Área florestal $\left(\mathrm{N}_{\mathrm{t}}\right)$ & ha & 14500 \\
\hline Área inicial de agricultura $\left(\mathrm{N}_{\mathrm{a}}\right)$ & ha & 1890,2 \\
\hline Custo de conversão de miombo & $\$$ por $\mathrm{m}^{3}$ & 0 \\
\hline Salário anual (w) & $\$$ por ano & 303,72 \\
\hline Preço médio da árvore de miombo em pé $\left(\mathrm{P}_{\text {ave }}\right)$ & $\$ / \mathrm{m}^{3}$ & 15,40 \\
\hline Preço de médio/ combustíveis lenhosos $\left(\mathrm{P}_{\mathrm{fw}}\right)$ & $\$ / \mathrm{m}^{3}$ & 3,06 \\
\hline Preço de estacas $\left(\mathrm{P}_{\mathrm{po}}\right)$ & $\$ / \mathrm{m}^{3}$ & 5 \\
\hline Compra de insumos & & 0 \\
\hline Regeneração (s) & & 0,012 \\
\hline Receita da agricultura $\left(\mathrm{P}_{\mathrm{a}}\right)$ & $\$ /$ ha & 253,66 \\
\hline Rendimento de subsistência $\left(\mathrm{I}_{\mathrm{h}}\right)$ & $\$$ & 734561 \\
\hline Taxa de sobrevivência & & 0,92 \\
\hline 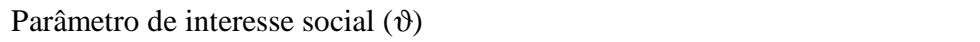 & & 0 ou 1 \\
\hline Mão de obra total potencial do setor familiar & Homens-dia & 986 \\
\hline Total de homens-dia na comunidade & Homens-dia & 716822 \\
\hline Parâmetro da função de custo da mão de obra (v) & & 0,5 \\
\hline Volume em pé de madeira $\left(\mathrm{K}_{\mathrm{t}}\right)$ & $\mathrm{m}^{3} / \mathrm{ha}$ & 47 \\
\hline Número de habitantes & & 4362 \\
\hline Custo de exploração florestal do setor privado & $\$ / \mathrm{m}^{3}$ & 7,8 \\
\hline $\begin{array}{l}\text { Parâmetro de eficiência para mão de obra para exploração florestal pelo setor } \\
\text { comercial }\left(\mathrm{q}_{\mathrm{c}}\right)\end{array}$ & & 0,083 \\
\hline Área potencial para exploração comercial & ha & 1234 \\
\hline
\end{tabular}

\section{RESULTADOS E DISCUSSÃO}

Esta seção analisa como as alternativas de regime de manejo influenciam os benefícios provenientes dos intervenientes e como estes se transformam em volumes de produtos explorados, emprego da mão de obra nas atividades na floresta de miombo e o estado dos recursos florestais. Sabendo que atualmente os recursos da floresta de miombo são principalmente explorados sob regimes de acesso livre, o modelo não cooperativo será usado como ponto de referência na comparação com os regimes alternativos de manejo.

\section{Impacto potencial nos benefícios líquidos dos intervenientes}

O efeito do regime de manejo nos benefícios totais descontados das atividades de miombo varia em termos de quantidade e distribuição entre os dois setores (Tabela 2). As diferenças nos benefícios 
líquidos, descontados entre os dois setores, reflete a diferença na eficiência de exploração e no valor de mercado dos produtos explorados.

Tabela 2. Efeito potencial dos regimes de manejo sobre os benefícios líquidos descontados dos intervenientes pela venda de produtos madeireiros e não madeireiros de miombo ('000 US\$) ${ }^{1}$.

Table 2. Effect of management regime on Net Discounted Benefits of stakeholders from sale of miombo wood and NTFPS products ('000 US \$) ${ }^{1}$.

\begin{tabular}{lccc}
\hline \multirow{2}{*}{ Regime de manejo } & \multicolumn{2}{c}{ Setor } & \multirow{2}{*}{ Total } \\
\cline { 2 - 3 } & Privado & Familiar & \\
\hline Centralizado com interesses sociais & $3578,95(3)$ & $410,33(1)$ & $3989,28(3)$ \\
Centralizado com interesses ambientais & $3227,31(4)$ & $21,45(5)$ & $3248,76(4)$ \\
Centralizado com interesses sociais e ambientais & $2877,04(5)$ & $272,77(2)$ & $3149,81(5)$ \\
Cooperativo & $4206,65(2)$ & $228,65(3)$ & $4435,30(2)$ \\
Não cooperativo & $5752,07(1)$ & $87,59(4)$ & $5839,66(1)$ \\
\hline${ }^{1}$ valores entre parênteses são os ordenamentos por ordem crescente dos regimes de manejo em termos de benefícios derivados \\
pelos intervenientes.
\end{tabular}

(a) O setor privado

Os mais altos benefícios líquidos descontados para o setor privado são obtidos sob o regime de manejo não cooperativo. Como o regime de manejo não cooperativo é o mais benéfico para o setor privado, este setor não estará interessado na intervenção do governo ou cooperação com o governo ou comunidades locais a não ser que os benefícios intangíveis e as multas por não cooperar excedam os benefícios adicionais provenientes da cooperação. O regime cooperativo (manejo comunitário) foi o segundo melhor, seguido pelo regime centralizado incorporando interesses sociais. Assim sendo, se o objetivo for promover o envolvimento do setor privado no manejo e uso da floresta de miombo, o regime de não cooperação (regime de acesso livre) ou status quo é o mais apropriado para o setor privado.

(b) O setor familiar

O regime de manejo que permite obter os mais altos benefícios líquidos descontados a partir de atividades florestais para o setor familiar é o regime centralizado incorporando interesses ambientais. Os benefícios obtidos pelo setor familiar foi $300 \%$ superior aos obtidos pelo regime não cooperativo. $\mathrm{O}$ regime centralizado incorporando interesses sociais e ambientais é a segunda melhor opção, seguido do regime cooperativo. A opção de manejo centralizada com interesses ambientais é o regime com menos benefícios monetários. Quando combinados os benefícios da agricultura e da floresta (Tabela 3), a mesma ordem de importância para os regimes de manejo é mantida.

Os resultados sugerem que se os órgãos de tomada de decisão ou os planificadores rurais colocarem muito peso no bem-estar do setor familiar rural, então o regime de manejo centralizado com interesses sociais ou sociais e ambientais aparece como mais apropriado como regime de manejo das florestas de miombo, seguido do regime cooperativo. O regime não cooperativo ou status quo apresentase como muito desvantajoso para as comunidades locais.

Tabela 3. Efeito das alternativas de manejo sobre os benefícios líquidos descontados totais e benefícios líquidos anuais per capita do setor familiar ${ }^{1}$.

Table 3. Effect of management regime on total net discounted benefits and annual net discounted benefits per capita of stakeholders.

\begin{tabular}{lcc}
\hline Regime de manejo & $\begin{array}{c}\text { Benefícios totais em US } \\
\mathbf{\$} \mathbf{0 0 0}\end{array}$ & $\begin{array}{c}\text { Benefícios líquidos anuais per } \\
\text { capita US\$ }\end{array}$ \\
\hline Centralizado com precauções sociais & $13100,20(1)$ & $60,07(1)$ \\
Centralizado com precauções ambientais & $12024,80(5)$ & $55,13(5)$ \\
Centralizado com precauções sociais e ambientais & $12621,50(2)$ & $57,87(2)$ \\
Cooperativo & $12604,20(3)$ & $57,79(3)$ \\
Não cooperativo & $12273,60(4)$ & $56,28(4)$ \\
\hline
\end{tabular}

${ }^{1}$ valores entre parênteses são os ordenamentos (rankings) dos regimes de manejo em termos de benefícios provenientes dos intervenientes. 
(c) A combinação dos setores: familiar e privado

Como grande parte dos benefícios pertence ao setor privado, os benefícios totais para os dois setores (privado e familiar) seguem a tendência dos benefícios do setor privado (Tabela 2). Para ambos os setores, se o objetivo for maximizar os benefícios líquidos descontados da exploração de produtos madeireiros da floresta de miombo sem considerar a distribuição entre os dois setores, o regime não cooperativo ou status quo será o mais atrativo, seguido pelo regime cooperativo e o centralizado com interesses sociais. O regime centralizado incorporando interesses sociais e ambientais providencia o menor potencial de benefícios líquidos descontados a partir da exploração de produtos madeireiros de miombo.

\section{Impacto potencial de diferentes regimes de manejo nos recursos florestais}

Os direitos de propriedade incompletos, práticas agrícolas inadequadas e falhas nas políticas na internalização das externalidades provenientes da exploração de recursos florestais têm contribuído para altas taxas de desflorestamento e degradação das terras. A fraca capacidade de institucionalização para aplicação da legislação existente para o uso dos recursos florestais tem conduzido a utilização/exploração dos recursos sob regimes não cooperativos. A tabela 4 apresenta um sumário do impacto potencial de diferentes regimes de manejo na área relativa da floresta de miombo e na área relativa convertida para agricultura usando o regime de manejo não cooperativo ou quo status como referência. A área da floresta em pé no fim do período de simulação (relativamente ao regime não cooperativo) é maior sob o regime centralizado com interesses ambientais, seguido pelo regime centralizado com interesses sociais e ambientais, regime cooperativo, regime centralizado incorporando benefícios sociais e por último o regime não cooperativo.

Tabela 4. Áreas relativas (\%) de floresta de miombo em pé e convertida para agricultura ${ }^{1}$.

Table 4. Relative area (\$) of standing miombo and converted to agriculture ${ }^{1}$.

\begin{tabular}{lcc}
\hline Regime de manejo & $\begin{array}{c}\text { Área relativa de floresta de } \\
\text { miombo em pé (\%) }\end{array}$ & $\begin{array}{c}\text { Área relativa convertida para } \\
\text { agricultura (\%) }\end{array}$ \\
\hline Centralizado com precauções sociais & $141,19(4)$ & $385,06(1)$ \\
Centralizado com precauções ambientais & $193,85(1)$ & $10,02(5)$ \\
Centralizado com precauções sociais e & $175,03(2)$ & $221,06(2)$ \\
ambientais & $151,10(3)$ & $202,74(3)$ \\
Cooperativo & $100,00(5)$ & $100,00(4)$ \\
Não cooperativo & & \\
' O regime de manejo não cooperativo é usado como base de comparação e os valores entre parênteses são indicativos da ordem de \\
prioridade.
\end{tabular}

O desflorestamento é alto sob o regime não cooperativo. No fim do período de simulação, cerca de $20 \%$ da área inicial mantém-se florestada. Como esperado, o regime centralizado com interesses ambientais leva-nos a menor desmatamento, embora seja a opção menos atrativa para os setores privado e familiar em termos de benefícios.

Em termos de benefícios para os setores privado e familiar, os regimes centralizados apresentamse mais favoráveis. Eles também se apresentam como escolha razoável quando é dada alguma consideração em relação à área relativa da floresta de miombo em pé. Todavia, com exceção do regime centralizado com interesses ambientais, as outras duas variantes do regime centralizado (social e social + meio ambiente) apresentam-se associadas com uma área relativa maior de floresta convertida para agricultura. Mas isso é o preço ou termo de troca para melhorar os benefícios totais do setor familiar.

\section{CONCLUSÕES}

- Os resultados mostram que melhorias no bem-estar e na conservação dos recursos podem ser alcançados com melhores práticas de manejo. Os resultados mostram que os regimes de manejo centralizados incorporando interesses sociais ou ambientais e sociais têm potencial, promovendo valores mais altos de benefícios para o setor familiar que o regime não cooperativo. $\mathrm{O}$ regime centralizado com interesses sociais tem potencial para melhorar os benefícios líquidos descontados a partir de produtos madeireiros em cerca de $300 \%$ relativamente ao regime não cooperativo. No caso 
do regime centralizado com interesses sociais e ambientais, há um incremento de mais de $200 \%$. Isso quer dizer que o regime centralizado com interesses sociais ou com interesses sociais e ambientais têm potencial para melhorar o bem-estar das comunidades rurais e encorajar a conservação florestal, mas somente se esses benefícios fossem atualmente sentidos ao nível das comunidades.

- Os resultados para o regime de manejo cooperativo (manejo comunitário) mostram que ambos os setores, privado e comunidades locais, podem se beneficiar desse regime. Embora o setor privado perca alguns benefícios diretos em favor do setor familiar, como se indicou na hipótese, os custos poupados pela redução de conflitos entre os dois setores podem induzir essa cooperação. No caso de Moçambique, onde a lei da terra permite que as comunidades possam entrar em sociedades com o setor privado para o manejo e uso dos recursos florestais, os resultados mostram que essa cooperação é potencialmente benéfica para as comunidades locais se for devidamente implementada.

\section{AGRADECIMENTOS}

Este artigo foi baseado num estudo realizado com apoio financeiro da União Europeia em favor das florestas tropicais nos países em desenvolvimento com base na linha orçamental (b7-6201) e do Centro Internacional de Investigação Florestal (CIFOR). Os autores agradecem esse apoio e são os únicos responsáveis por todas as opiniões expressas neste documento, as quais não necessariamente refletem as da União Europeia e do CIFOR.

\section{REFERÊNCIAS}

ANSTEY, S. Necessarily vague: the political economy of community conservation in Mozambique. In: HULME, D.; MURPHREE, M.; CURREY, J. (Ed.) African wildlife and African livelihoods: the promise and performance of community conservation. Oxford, Great Britain, 2001. p. 74 - 87.

CARDENAS, J. C.; STRANLAND, J.; WILLIS, C. Local environmental control and institutional crowding-out. World Development, v. 28, n. 10, p. 1719 - 1733, 2000.

FALCÃO, M. P.; SUMAILA, R. U.; GRUNDY, I. M.; GELDENHUYS, C. J. The impact of policy on resource use in Mozambique: a case study of Savane. Silva Lusitana, v. 15, n. 1, 2007.

FLÅM, S. D. Path to constrained Nash equilibria. Applied Mathematics and Optimisation, v. 27, p. $275-289,1993$.

FOREST TREES AND PEOPLE NEWSLETTER Approaches to wildlife development: lessons from Zambia, Zimbabwe. Forests, Trees and People Newsletter, v. 13, p. 23 - 29, 1991.

KANT, S.; NAUTIYAL, J. C. Sustainable management through bargaining: a bilateral monopoly gaming approach. Forest Ecology and Management, v. 65, p. 251 - 264, 1993.

McCARTHY, N. An economic analysis of the effects of production risk on the use and management of common-pool rangelands. In: McCARTHY, N.; SWALLOW, B.; KIRK, M.; HAZELL, P. (Eds.) Property rights, risk and livestock development in Africa. IFPRI, 2000.

MURPHREE, M. W. Congruent objectives, competing interests and strategic compromise: concepts and process in the evaluation of Zimbabwe's CAMPFIRE programme. Community conservation in Africa: principles and comparative practice. University of Manchester, 1997. (Paper n. 2).

NHANTUMBO, I. The new resource tenure framework in Mozambique: does it really give the tenancy to the rural community? In: CONFERENCE OF THE INTERNATIONAL ASSOCIATION FOR THE STUDY OF COMMON PROPERTY, 8., 2000, USA. Abstracts of... USA: IASCP, 2000.

SUMAILA, U. R. Irreversible capital investment in a two-stage bimatrix fishery game model. Marine Resource Economics, v. 10, n. 3, p. 263 - 283, 1995. 
SUMAILA, U. R.; ANGELSEN, A.; KOWERO, G. A system dynamics model for management of miombo woodlands. In: Modelling methods for policy analysis in miombo woodlands. Bogor: CIFOR, 2001. p. 17 - 30. (Occasional Paper n. 35).

WHITE, B. A review of the economics of biological natural resources. Journal of Agricultural Economics. v. 51, p. 419 - 462, 2000.

WILY, L.; MBAYA, S. Land, people and forests in eastern and southern Africa at the beginning of the $21^{\text {st }}$ century: the impact of land relations on the role of communities in forest future. IUCN Eastern Africa Regional office, 2001.313 p. 